\title{
Performance Analysis of Full-Duplex Decode-and-Forward Two-Way Relay System
}

\author{
Ganning He, Lixin Li, Jie Fan, Zihe Zhang, Xu Li, Zhibin Xu \\ School of Electronics and Information, \\ Northwestern Polytechnical University, Xi'an, China \\ E-mail:heganning@sina.com,\{lilixin,zhanghuisheng\}@nwpu.edu.cn
}

\begin{abstract}
This paper considers a full-duplex two-way relaying (FD-TWR) system, where two terminals transmit and receive simultaneously with the help of a full-duplex relay node. We propose the transmission scheme for decode-and-forward (DF) protocol using the differential binary phase-shift keying (DBPSK) modulation to analyse the bit error ratio (BER), which do not need the channel state information (CSI). We consider the residual self-interference (RSI) in relay and source. By using the maximum a posteriori (MAP) and maximum likelihood (ML) decoder at the relay and the source node, respectively, we obtain the symbols. The simulation results show that the full-duplex decode-and-forward two-way relaying (F2DF-TWR) system can achieve the bit error performance of the half-duplex decode-and-forward two-way relaying (H2DF-TWR) system.

Keywords: full-duplex; two-way; decode-and-forward; residual self-interference; non-coherent maximum likelihood.
\end{abstract}

\section{Introduction}

In conventional cooperative communication systems, all the nodes operate in the half-duplex (HD) mode, which have to transmit and receive signals in different frequency bands and/or different time slots. Traditional half-duplex one-way relay system requires four time slots to exchange one frame, which leads to a loss in spectral efficiency [1]. In order to improve the spectral efficiency, TWR, two-path successive relaying and the FD are proposed. TWR is applicable only to bidirectional communication, and two-path successive relaying is a promising protocol [2], but suffer from severe inter-relay interference. The FD mode can efficiently improve the spectral efficiency by transmitting and receiving signals in the same time slot and frequency band. However, since the FD mode suffer from the strong RSI, it was not considered for a long time. With the development in digital and analog domain self-interference cancellation (SIC) technologies [3], the SI can be greatly eliminated, so the FD mode draws our attention [4].

Most of the previous cooperation schemes on FDF-TWR system mainly focus on the outage probabilities [5] and spectral-efficient [6]. And also a lot of works assume CSI is known at the receiver [6], [7], which is hard to satisfy in practice. But long-term CSI is easier to get and requires less overhead [8]. Therefore, this motivates the schemes using non-coherent modulation that do not require instant CSI. [9] proposes new schemes for AF and DF protocols where do not need the CSI known and derives ML detectors for both protocols. [10] proposes a DF TWR scheme and a denoise-and-forward (DNF) TWR scheme using non-coherent multiple differential phaseshift keying modulation in the half-duplex system, which do not need CSI. [11] uses the DNF scheme with DBPSK modulation to recover the spectral efficiency loss caused by the half-duplex relay, which do not need CSI either. Motivated by these, we try to combine the non-coherent modulation with FD wireless communication to analyse the BER.

In this paper, we study the F2DF-TWR scheme with DBPSK to analyse the BER, which do not need instantaneous CSI. And we consider the RSI. The simulation results show that the proposed FDF-TWR system can achieve the bit error performance of the H2DF-TWR system.

Notations: matrices and variables are denoted by bold capital and lower-case letters, respectively; $(\cdot)^{*}$, $(\cdot)^{T}$ and $(\cdot)^{H}$ stand for conjugate, transpose, and conjugate transpose, respectively.

\section{System model}

In this paper, we investigate a FD-TWR system, as shown in Figure 2. The typical three-node model is applied to the FD enabled TWR system, which comprises two terminal nodes $A, B$ and one relay node $R$ with transmitting power $P_{A}, P_{B}$ and $P_{C}$, respectively. Here, we assume $P_{A}=P_{B}=P_{C}=P$. Each node has two 
antennas that one is for transmitting and another is used for receiving. And the RSI is taken into consideration. The physical-layer network coding (PNC) based on DF TWR [12], as shown in Figure 1, only needs two time slots to complete the bidirectional transmission. We assume the time, phase and carrier of all transmitted signals are synchronized.

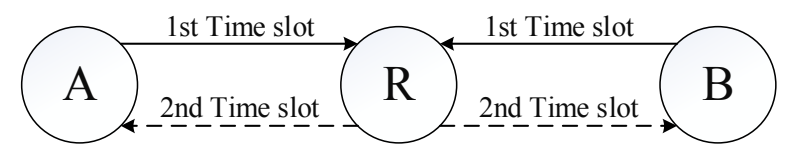

Figure 1. Bi-directional information exchange using PNC

There are $L$ frames to be exchanged at the source nodes $A$ and $B$, and it needs $L+1$ time slots to transmit the $2 L$ frames. The signals to be transmitted by $A$ and $B$ are denoted by $c_{A}^{t}(n)$ and $c_{B}^{t}(n)$, respectively, and the signals decoded and to be sent in the relay are denoted by $c_{R}^{t}(n)$, where $n$ denotes the symbol index in one frame, $t$ denotes the time slot.

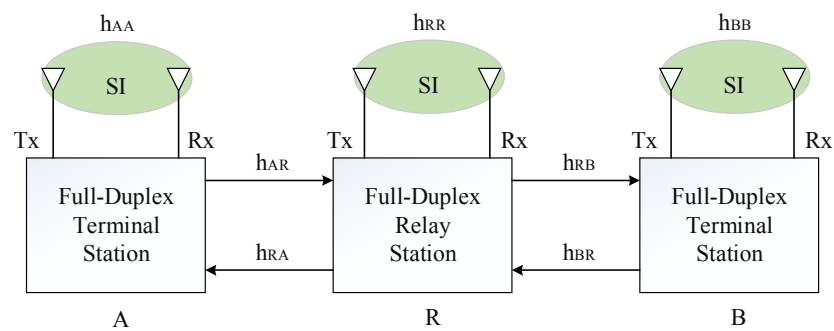

Figure 2. The proposed full-duplex decode-and-forward two-way relay system

According to the Figure 2, there are the $A \rightarrow R$ channel, the $B \rightarrow R$ channel, the $R \rightarrow A$ channel, the $R \rightarrow B$ channel, and the RSI channels at $A, B$ and $R$ in the system. And the corresponding channel fading coefficients are $h_{A R}, h_{B R}, h_{R A}, h_{R B}, h_{A A}, h_{B B}$ and $h_{R R}$.

To better express the this paper, we make the following assumptions: (1) All the channel coefficients remain static over one time slot; (2) The channels between two nodes and the RSI channels are subjected to Rayleigh fading; (3) The CSI is known at the node $A, B$ and $R$, i.e., $h_{A R}^{t} \sim \mathrm{CN}\left(0, \sigma_{A R}^{2}\right), h_{B R}^{t} \sim \mathrm{CN}\left(0, \sigma_{B R}^{2}\right)$, $h_{R A}^{t} \sim \mathrm{CN}\left(0, \sigma_{R A}^{2}\right), h_{R B}^{t} \sim \mathrm{CN}\left(0, \sigma_{R B}^{2}\right), h_{A A}^{t} \sim \mathrm{CN}\left(0, \sigma_{A A}^{2}\right), h_{B B}^{t} \sim \mathrm{CN}\left(0, \sigma_{B B}^{2}\right)$ and $h_{R R}^{t} \sim \mathrm{CN}\left(0, \sigma_{R R}^{2}\right)$, where $\sigma_{A R}^{2}, \sigma_{B R}^{2}, \sigma_{R A}^{2}, \sigma_{R B}^{2}, \sigma_{A A}^{2}, \sigma_{B B}^{2}$ and $\sigma_{R R}^{2}$ denote the corresponding channel gain, and $\sigma_{A R}^{2}=\sigma_{B R}^{2}=\sigma_{R A}^{2}=\sigma_{R B}^{2}=\sigma^{2}$, $\sigma_{A A}^{2}=\sigma_{B B}^{2}=\sigma_{R R}^{2}=\sigma_{R S I}^{2}$; (4) There is no direct link between A and B due to the strong shadowing effect and the long communication distance; (5) The noises $n_{A}, n_{B}$, and $n_{R}$ over the node $A, B$ and $R$ are independently and identically distributed (i.i.d) Gaussian noises with zero mean and variance of $\sigma_{n}^{2}$, i.e., $n_{i} \sim \mathrm{CN}\left(0, \sigma_{n}^{2}\right), i=\{A, B, R\}$.

In the $t$ th $(t=1,2, \cdots, L)$ time slot, the two source nodes $A$ and $B$ firstly generate the $t$ th frame BPSK symbols $c_{A}^{t}(n) \in\{1,-1\}$ and $c_{B}^{t}(n) \in\{1,-1\}$ respectively. Then nodes $A$ and $B$ generate the DBPSK symbols $x_{A}^{1}=c_{A}^{t}(n) \times x_{A}^{t}(n)$ and $x_{B}^{t}(n+1)=c_{B}^{t}(n) \times x_{B}^{t}(n)$ respectively, with $x_{A}^{t}(1)=1$ and $x_{B}^{t}(1)=1$ being the reference symbol. Finally, $A$ and $B$ transmit $x_{A}^{t}(n)$ and $x_{B}^{t}(n)$ to $R$, respectively. And in the $t$ th $(t=2,3, \cdots, L+1)$ time slot, $R$ broadcast $x_{R}^{t}(n)$ to $A$ and $B$, where $x_{R}^{t}(n+1)=c_{R}^{t}(n) \times x_{R}^{t}(n)$.

\section{FDF-TWR Scheme}

Based on the system model of FDF-TWR, as demonstrated in the Figure 2 in section 2, we will discuss the signals process in this section.

In the 1 st time slot, the two source nodes transmit their first frame symbols to $R$, and the signals received at $R$ can be expressed as 


$$
y_{R}^{1}(n)=\sqrt{P_{A}} h_{A R}^{1} x_{A}^{1}(n)+\sqrt{P_{B}} h_{B R}^{1} x_{B}^{1}(n)+\mathrm{z}_{R}^{1}(n)
$$

In the $t$ th $(t=2,3, \cdots, L)$ time slot, the two source nodes transmit their $t$ th frame symbols to $R$, and simultaneously, $R$ forwards the symbols received and decoded in the previous time slot. The signals received at $A, B$ and $R$ can be respectively expressed as

$$
\begin{gathered}
y_{R}^{t}(n)=\sqrt{P_{A}} h_{A R}^{t} x_{A}^{t}(n)+\sqrt{P_{B}} h_{B R}^{t} x_{B}^{t}(n)+\sqrt{P_{R}} h_{R R}^{t} x_{R}^{t}(n)+\mathrm{z}_{R}^{t}(n) \\
y_{A}^{t}(n)=\sqrt{P_{A}} h_{A A}^{t} x_{A}^{t}(n)+\sqrt{P_{R}} h_{R A}^{t} x_{R}^{t}(n)+\mathrm{z}_{A}^{t}(n) \\
y_{B}^{t}(n)=\sqrt{P_{B}} h_{B B}^{t} x_{B}^{t}(n)+\sqrt{P_{R}} h_{R B}^{t} x_{R}^{t}(n)+\mathrm{z}_{B}^{t}(n)
\end{gathered}
$$

In the $(L+1)$ th time slot, $R$ forwards the symbols received and decoded in the $L$ th time slot, and the signals received at $A$ and $B$ can be respectively expressed as

$$
\begin{aligned}
& y_{A}^{L+1}(n)=\sqrt{P_{R}} h_{R A}^{L+1} x_{R}^{L+1}(n)+\mathrm{z}_{A}^{L+1}(n) \\
& y_{B}^{L+1}(n)=\sqrt{P_{R}} h_{R B}^{L+1} x_{R}^{L+1}(n)+\mathrm{z}_{B}^{L+1}(n)
\end{aligned}
$$

Table 1 and Table 2 summary the transmitted and received signals at each node during one time slot, respectively.

Table 1. Transmitted signals at each node

\begin{tabular}{|c|c|c|c|c|c|c|}
\hline Time slot & 1 & 2 & $\ldots$ & $\mathrm{L}-1$ & $\mathrm{~L}$ & $\mathrm{~L}+1$ \\
\hline $\mathrm{A}$ & $x_{A}^{1}$ & $x_{A}^{2}$ & $\ldots$ & $x_{A}^{L-1}$ & $x_{A}^{L}$ & \\
\hline $\mathrm{B}$ & $x_{B}^{1}$ & $x_{B}^{2}$ & $\ldots$ & $x_{B}^{L-1}$ & $x_{B}^{L}$ & \\
\hline $\mathrm{R}$ & & $x_{R}^{2}$ & $\ldots$ & $x_{R}^{L-1}$ & $x_{R}^{L}$ & $x_{R}^{L+1}$ \\
\hline
\end{tabular}

Table 2. Received signals at each node

\begin{tabular}{|c|c|c|c|c|c|c|}
\hline Time slot & 1 & 2 & $\ldots$ & $\mathrm{L}-1$ & $\mathrm{~L}$ & $\mathrm{~L}+1$ \\
\hline $\mathrm{A}$ & & $y_{A}^{2}$ & $\ldots$ & $y_{A}^{L-1}$ & $y_{A}^{L}$ & $y_{A}^{L+1}$ \\
\hline $\mathrm{B}$ & & $y_{B}^{2}$ & $\ldots$ & $y_{B}^{L-1}$ & $y_{B}^{L}$ & $y_{B}^{L+1}$ \\
\hline $\mathrm{R}$ & $y_{R}^{1}$ & $y_{R}^{2}$ & $\ldots$ & $y_{R}^{L-1}$ & $y_{R}^{L}$ & \\
\hline
\end{tabular}

\subsection{PNC mapping}

\begin{tabular}{|c|c|c|c|c|c|c|}
\hline \multirow{2}{*}{\multicolumn{4}{|c|}{ Modulation mapping at $A$ and $B$}} & \multicolumn{3}{|c|}{ Demodulation mapping at $R$} \\
\hline & & & & \multirow[t]{3}{*}{ Input } & \multirow{2}{*}{\multicolumn{2}{|c|}{$\begin{array}{c}\text { Output } \\
\text { Modulation mapping at } \\
R\end{array}$}} \\
\hline \multirow{2}{*}{\multicolumn{2}{|c|}{ Input }} & \multirow{2}{*}{\multicolumn{2}{|c|}{ Output }} & & & \\
\hline & & & & & Input & Output \\
\hline$s_{A}^{t}$ & $s_{B}^{t}$ & $c_{A}^{t}$ & $c_{B}^{t}$ & $c_{A}^{t}+c_{B}^{t}$ & $s_{A}^{t} \oplus s_{B}^{t}$ & $c_{R}^{t}$ \\
\hline 1 & 1 & -1 & -1 & -2 & 0 & 1 \\
\hline 0 & 1 & 1 & -1 & 0 & 1 & -1 \\
\hline 1 & 0 & -1 & 1 & 0 & 1 & -1 \\
\hline 0 & 0 & 1 & 1 & 2 & 0 & 1 \\
\hline
\end{tabular}

The realization of the PNC scheme based on DF relay is closely related to the demodulation mapping. As shown in Figure 1, the nodes $A$ and $B$ transmit the BPSK-modulated signal to the relay node $R$ at the same time slot, the received superimposed signal is demodulated at $R$.

Table 3. PNC Mapping: modulation mapping at $A$ and $B$; demodulation and modulation mappings at $R$

The BPSK modulation-based PNC demodulation mapping scheme [12] is shown in Table 3. The relay node $R$ transmits the signals $c_{R}^{t}$ to the nodes $A$ and $B$ in the second time slot, according to the modulation/demodulation mapping scheme proposed in Table 3. Nodes $A$ and $B$ carry on BPSK demodulation to the received data again, can get the $s_{A}^{t} \oplus s_{B}^{t}$, then do the exclusive-OR operation with the information that they have sent respectively, complete the information exchange. 


\subsection{Decode-and-forward}

At the relay node $R$, we define $\boldsymbol{y}_{R}^{t}(n)=\left[\begin{array}{ll}y_{R}^{t}(n+1) & y_{R}^{t}(n)\end{array}\right]^{H}(t=2,3, \cdots, L+1)$. According to (2), we have the conditional probability density function (PDF) of $\boldsymbol{y}_{R}^{t}(n)$ given $c_{A}^{t}(n), c_{B}^{t}(n)$ and $c_{R}^{t}(n)$, i.e.

$$
f\left(\boldsymbol{y}_{R}^{t}(n) \mid c_{A}^{t}(n), c_{B}^{t}(n), c_{R}^{t}(n)\right)=\frac{1}{\pi^{2}\left|\boldsymbol{K}_{R}^{t}(n)\right|} \exp \left\{-\boldsymbol{y}_{R}^{t}(n)^{H} \boldsymbol{K}_{R}^{t}(n)^{-1} \boldsymbol{y}_{R}^{t}(n)\right\}
$$

where $\boldsymbol{K}_{R}^{t}(n)$ denotes the covariance matrix can be expressed as

$$
\boldsymbol{K}_{R}^{t}(n)=\left[\begin{array}{cc}
P_{A} \sigma_{A R}^{2}+P_{B} \sigma_{B R}^{2}+P_{R} \sigma_{R R}^{2}+\sigma_{n}^{2} & P_{A} \sigma_{A R}^{2} c_{A}^{t}(n)+P_{B} \sigma_{B R}^{2} c_{B}^{t}(n)+P_{R} \sigma_{R R}^{2} c_{R}^{t}(n) \\
P_{A} \sigma_{A R}^{2} c_{A}^{t}(n)^{*}+P_{B} \sigma_{B R}^{2} c_{B}^{t}(n)^{*}+P_{R} \sigma_{R R}^{2} c_{R}^{t}(n)^{*} & P_{A} \sigma_{A R}^{2}+P_{B} \sigma_{B R}^{2}+P_{R} \sigma_{R R}^{2}+\sigma_{n}^{2}
\end{array}\right]
$$

Since $c_{R}^{t}(n)$ are known at $R$, and we let $c_{A B}^{t}(n)=c_{A}^{t}(n)+c_{B}^{t}(n)$, so (7) and (8) can be rewrite as

$$
\begin{gathered}
f\left(\boldsymbol{y}_{R}^{t}(n) \mid c_{A B}^{t}(n)\right)=\frac{1}{\pi^{2}\left|\boldsymbol{K}_{R}^{t}(n)\right|} \exp \left\{-\boldsymbol{y}_{R}^{t}(n)^{H} \boldsymbol{K}_{R}^{t}(n)^{-1} \boldsymbol{y}_{R}^{t}(n)\right\} \\
\boldsymbol{K}_{R}^{t}(n)=\left[\begin{array}{cc}
2 P \sigma^{2}+P \sigma_{R S I}^{2}+\sigma_{n}^{2} & P \sigma^{2} c_{A B}^{t}(n)+P \sigma_{R S I}^{2} c_{R}^{t}(n) \\
P \sigma^{2} c_{A B}^{t}(n)^{*}+P \sigma_{R S I}^{2} c_{R}^{t}(n)^{*} & 2 P \sigma^{2}+P \sigma_{R S I}^{2}+\sigma_{n}^{2}
\end{array}\right]
\end{gathered}
$$

As several $c_{A}^{t}(n)$ and $c_{B}^{t}(n)$ pairs may result the same value of $c_{A B}^{t}(n)=c_{A}^{t}(n)+c_{B}^{t}(n)$, we use the MAP detector by maximizing $f\left(c_{A B}^{t}(n) \mid \boldsymbol{y}_{R}^{t}(n)\right)=\frac{f\left(\boldsymbol{y}_{R}^{t}(n) \mid c_{A B}^{t}(n)\right) f\left(c_{A B}^{t}(n)\right)}{f\left(\boldsymbol{y}_{R}^{t}(n)\right)}$. Because $f\left(\boldsymbol{y}_{R}^{t}(n)\right)$ do not affect the result, we can obtain

$$
\hat{c}_{A B}^{t}(n)=\arg \max f\left(\boldsymbol{y}_{R}^{t}(n) \mid c_{A B}^{t}(n)\right) \cdot f\left(c_{A B}^{t}(n)\right)
$$

According to the demodulation and modulation mappings at $R$, the corresponding $c_{R}^{t}$ will be used as the forwarded symbols of the relay in the next time slot, i.e., $c_{R}^{t+1}(n)$.

Similarly, at the source nodes $A$ and $B$ (we only talk about the node $B$, because it is symmetric), we define $\boldsymbol{y}_{A}^{t}(n)=\left[\begin{array}{ll}y_{A}^{t}(n+1) & y_{A}^{t}(n)\end{array}\right]^{H}(t=2,3, \cdots, L+1)$ as well. According to (3), we have $\boldsymbol{y}_{A}^{t}(n) \sim \mathrm{CN}\left(0, \boldsymbol{K}_{A}^{t}(n)\right)$ given $c_{A}^{t}(n)$ and $c_{R}^{t}(n)$, where $\boldsymbol{K}_{A}^{t}(n)$ denotes the covariance matrix can be derived as

$$
\boldsymbol{K}_{A}^{t}(n)=\left[\begin{array}{cc}
P_{R} \sigma_{R A}^{2}+P_{A} \sigma_{A A}^{2}+\sigma^{2} & P_{R} \sigma_{R A}^{2} c_{R}^{t}(n)+P_{A} \sigma_{A A}^{2} c_{A}^{t}(n) \\
P_{R} \sigma_{R A}^{2} c_{R}^{t}(n)^{*}+P_{A} \sigma_{A A}^{2} c_{A}^{t}(n)^{*} & P_{R} \sigma_{R A}^{2}+P_{A} \sigma_{A A}^{2}+\sigma^{2}
\end{array}\right]
$$

The conditional probability density function (PDF) of $\boldsymbol{y}_{A}^{t}(n)$ given $c_{A}^{t}(n)$ and $c_{R}^{t}(n)$ is

$$
f\left(\boldsymbol{y}_{A}^{t}(n) \mid c_{A}^{t}(n), c_{R}^{t}(n)\right)=\frac{1}{\pi^{2}\left|\boldsymbol{K}_{A}^{t}(n)\right|} \exp \left\{-\boldsymbol{y}_{A}^{t}(n)^{H} \boldsymbol{K}_{A}^{t}(n)^{-1} \boldsymbol{y}_{A}^{t}(n)\right\}
$$

Since $c_{A}^{t}(n)$ are known at $R$, we can respectively rewrite (12) and (13) as

According to ML, we have

$$
\begin{gathered}
f\left(\boldsymbol{y}_{A}^{t}(n) \mid c_{R}^{t}(n)\right)=\frac{1}{\pi^{2}\left|\boldsymbol{K}_{A}^{t}(n)\right|} \exp \left\{-\boldsymbol{y}_{A}^{t}(n)^{H} \boldsymbol{K}_{A}^{t}(n)^{-1} \boldsymbol{y}_{A}^{t}(n)\right\} \\
\boldsymbol{K}_{A}^{t}(n)=\left[\begin{array}{cc}
P \sigma^{2}+P \sigma_{R S I}^{2}+\sigma_{n}^{2} & P \sigma^{2} c_{R}^{t}(n)+P \sigma_{R S I}^{2} c_{A}^{t}(n) \\
P \sigma^{2} c_{R}^{t}(n)^{*}+P \sigma_{R S I}^{2} c_{A}^{t}(n)^{*} & P \sigma^{2}+P \sigma_{R S I}^{2}+\sigma_{n}^{2}
\end{array}\right]
\end{gathered}
$$

$$
\hat{c}_{R}^{t}(n)=\arg \max f\left(\boldsymbol{y}_{R}^{t}(n) \mid c_{R}^{t}(n)\right)
$$

Therefore, we get decoding result $\hat{c}_{R}^{t}(n)$ for $c_{R}^{t}(n)$. Then according to the Table 3 , we get the corresponding $c_{A B}^{t}(n)=c_{A}^{t}(n)+c_{B}^{t}(n)$. Because $A$ knows $c_{A}^{t}(n)$, the signals $\hat{c}_{B}^{t}(n)$ transmitted from the other can be decoded by

$$
\hat{c}_{B}^{t}(n)=\hat{c}_{R}^{t}(n) \oplus c_{A}^{t-1}(n)
$$




\section{Simulation results}

In this section, we analyse the BER performance of the full-duplex decode-and-forward two-way-relay (F2DF-TWR) system under the influence of self-interference and we compare it with the half-duplex decodeand-forward two-way-relay (H2DF-TWR) system. Figure 3 shows when the SNR is 20 and $30 \mathrm{~dB}$, the BER versus $1 / \sigma_{R S I}^{2}$ curves will approach a value. Figure 4 shows the BER versus the SNR of the F2DF-TWR system with $1 / \sigma_{R S I}^{2}=30$ and the H2DF-TWR, we can see that the curves of the two is similar.

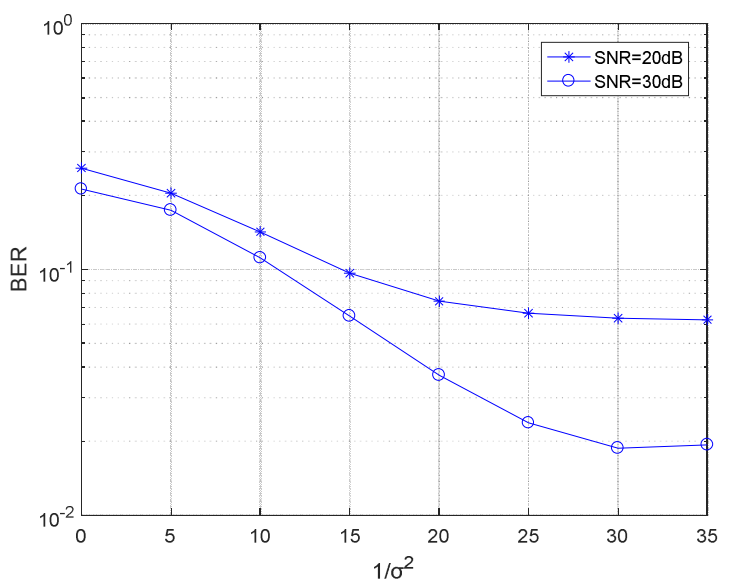

Figure 3. The BER versus $1 / \sigma_{R S I}^{2}$ curve of the F2DF-TWR

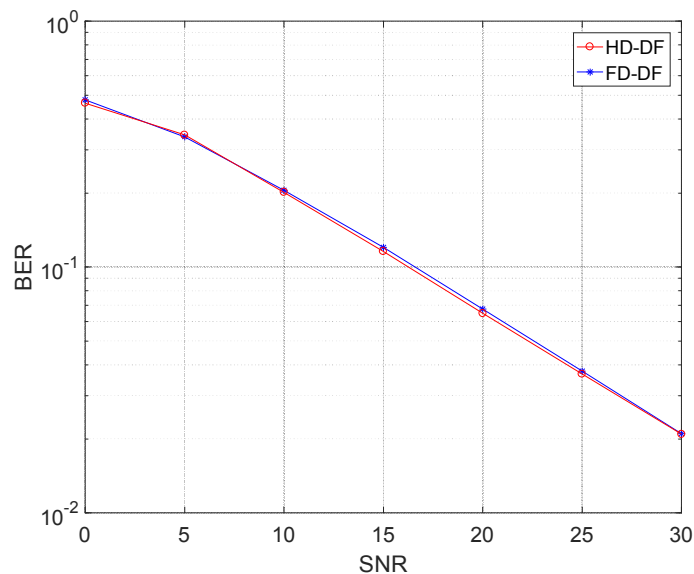

Figure 4. The BER comparison of F2DF-TWR with $1 / \sigma_{R S I}^{2}=30$ and H2DF-TWR

\section{Conclusion}

In this paper, we investigate a full-duplex decode-and-forward two-way relaying (F2DF-TWR) system. To effectively use the spectrum resources and improve the rate, we apply the PNC based DF. By using DBPSK with MAP and ML decoder at the relay and the source node, respectively, we can correctly obtain the information from the other. The simulation results show that the F2DF-TWR system can achieve a good BER performance with the RSI cancelled well.

\section{Acknowledgement}

This work is supported by Shaanxi Province natural science basic research plan surface project (No. 2016JM6062), by China Aerospace Science and Technology Corporation Aerospace Science and Technology 
Innovation Fund funded project, by the Seed Foundation of Innovation and Creation for Graduate Students in Northwestern Polytechnical University (No. Z2016106).

\section{References}

[1] B. Rankov and A. Wittneben. Spectral efficient protocols for half duplex fading relay channels. IEEE Journal on Selected Areas in Communications, 2007:379-389.

[2] W. Chen. Cao-sir: Channel aware ordered successive relaying. IEEE Trans. Wireless Commun, 2014:6513-6527.

[3] D. Korpi, L. Anttila, V. Syrjala, and M. valkama. Widely linear digital self-interference cancellation in directconversion full-duplex transceiver. IEEE Journal on Selected Areas in Communications, 2014:1674-1687.

[4] C. D. Melissa Duarte and A. Sabharwal. Experiment-driven characterization of full-duplex wireless systems. IEEE Trans. Wireless Commun, 2012:4296-4307.

[5] C. Li, Z. Chen, Y. Wang, Y. Yao, B. Xia. Outage Analysis of the Full-Duplex Decode-and-Forward Two-Way Relay System. IEEE Transactions on Vehicular Technology, 2016. DOI: 10.1109/TVT.2016.2610004.

[6] L. Li, C. Dong, L. Wang and L. Hanzo. Spectral-Efficient Bidirectional Decode-and-Forward Relaying for FullDuplex Communication. IEEE Transactions on Vehicular Technology, 2016:7010-7020.

[7] Cheng Li, Yafei Wang, Zhiyong Chen, Yao Yao and Bin Xia. Performance analysis of the full-duplex enabled decodeand-forward two-way relay system. 2016 IEEE International Conference on Communications Workshops (ICC). IEEE. 2016:559-564.

[8] L. Li, C. Dong, L. Wang and L. Hanzo. Spectral-Efficient Bidirectional Decode-and-Forward Relaying for FullDuplex Communication. IEEE Transactions on Vehicular Technology, 2016:7010-7020.

[9] T. Cui, F. Gao and C. Tellambura. Differential modulation for two-way wireless communications: a perspective of differential network coding at the physical layer. IEEE Transactions on Communications, 2009:2977-2987.

[10] J. Fan, L. Li, T. Bao and H. Zhang. Two-way relaying with differential MPSK modulation in virtual full duplexing system. 2015 IEEE/CIC International Conference on Communications in China (ICCC). IEEE. 2015:1-5.

[11] Jie Fan; Lixin Li; Huisheng Zhang; Wei Chen. Denoise-and-Forward Two-Path Successive Relaying with DBPSK Modulation. IEEE Wireless Communications Letters, 2016. DOI: 10.1109/LWC.2016.2629471.

[12] Zhang S, Liew S C, Lam P P K. Physical Layer Network Coding. Acm Mobicom, 2007:2166-2168. 